\title{
Curvature of Visual Space Under Vertical Eye Rotation: Implications for Spatial Vision and Visuomotor Control
}

\author{
J. Douglas Crawford, ${ }^{1,2}$ Denise Y. P. Henriques, ${ }^{1,2}$ and Tutis Vilis ${ }^{1,3}$ \\ ${ }^{1}$ Medical Research Council Group for Action and Perception and ${ }^{2}$ Centre for Vision Research and Departments of \\ Psychology and Biology, York University, Toronto, Ontario, Canada M3J 1P3, and ${ }^{3}$ Department of Physiology, University \\ of Western Ontario, London, Ontario, Canada N6A 5C1
}

Most models of spatial vision and visuomotor control reconstruct visual space by adding a vector representing the site of retinal stimulation to another vector representing gaze angle. However, this scheme fails to account for the curvatures in retinal projection produced by rotatory displacements in eye orientation. In particular, our simulations demonstrate that even simple vertical eye rotation changes the curvature of horizontal retinal projections with respect to eye-fixed retinal landmarks. We confirmed the existence of such curvatures by measuring target direction in eye coordinates in which the retinotopic representation of horizontally displaced targets curved obliquely as a function of vertical eye orientation. We then asked subjects to point (open loop) toward briefly flashed targets at various points along these lines of curvature. The vectoraddition model predicted errors in pointing trajectory as a

As we navigate through the visual world, our viewpoint is constantly changing, as are the spatial relationships between our eyes, head, and body (Howard, 1982). Thus, the brain must account for these different internal frames of reference to chart visual space (Hallet and Lightstone, 1976; Zee et al., 1976; Mays and Sparks, 1980; Zipser and Anderson, 1988; Flanders et al., 1992; Maunsell, 1995; Miller, 1996). Studies that have addressed this problem have generally considered the angle of gaze direction relative to the head, adding this to retinotopic representations in a vectorial manner to reconstruct visual space in head coordinates (Hallet and Lightstone, 1976; Zee et al., 1976; Mays and Sparks, 1980; Zipser and Anderson, 1988; Flanders et al., 1992; Miller, 1996; Bockisch and Miller, 1999). This model is generally assumed to hold for secondary (vertical and horizontal) eye positions, perhaps supplemented by additional mechanisms that might compensate for tilts of the retina that occur during pure torsional eye rotations (rotation about the line of site) (Howard, 1982; Mittelstaed, 1983; Wade and Curthoys, 1997) and the so-called "false torsion" that occurs at tertiary (oblique) eye positions (von Helmholtz, 1867; Haustein and Mittelstaedt, 1990).

Received Aug. 10, 1999; revised Jan. 10, 2000; accepted Jan. 10, 1999.

This work was supported by grants from the Canadian Natural Sciences and Engineering Research Council and Canadian Medical Research Council (MRC) to J.D.C. and T.V. D.Y.P.H. is supported by an E. A. Baker Foundation CNIB-MRC Doctoral Research Award. J.D.C. is supported by an MRC Scholarship. We thank Drs. I. Howard, L. Harris, D. Tweed, J. Cullen, K. Humphrey, and P. Medendorp for constructive criticism on this manuscript.

Correspondence should be addressed to Prof. J. D. Crawford, Department of Psychology, York University, 4700 Keele Street, Toronto, Ontario, Canada M3J 1P3. E-mail: jdc@yorku.ca.

Copyright (C) 2000 Society for Neuroscience $\quad 0270-6474 / 00 / 202360-09 \$ 15.00 / 0$ function of eye orientation. In contrast, with only minor exceptions, actual subjects showed no such errors, showing a complete neural compensation for the eye position-dependent geometry of retinal curvatures. Rather than bolstering the traditional model with additional corrective mechanisms for these nonlinear effects, we suggest that the complete geometry of retinal projection can be decoded through a single multiplicative comparison with three-dimensional eye orientation. Moreover, because the visuomotor transformation for pointing involves specific parietal and frontal cortical processes, our experiment implicates specific regions of cortex in such nonlinear transformations.

Key words: visuomotor; spatial vision; eye position; retina; three-dimensional; geometry; arm movement; pointing

One problem with this implicitly common view is that it fails to account for the complex three-dimensional (3-D) properties of retinal geometry (Liu and Schor, 1998) and its dependence on eye rotation (Crawford and Guitton, 1997). Contrary to intuitions drawn from translational geometry, rotatory displacements in eye orientation have a strong and complex influence on the final static pattern of retinal stimulation. For example, we have recently confirmed that the retinal projections at tertiary eye positions in Listing's plane must be compared with eye orientation (presumably in the brainstem) to generate accurate saccades that obey Listing's law (Klier and Crawford, 1998). However, a similar geometric analysis (Crawford and Guitton, 1997) can be used to demonstrate an even more fundamental principle that is independent of Listing's law; the retinal projections of earth-fixed horizontal lines should curve with respect to a horizontal arc fixed on the retina as a function of vertical eye position (see Theory). Such an effect would be so basic as to impact almost any aspect of spatial vision and visuomotor control.

To our knowledge, curvature of retinal space at secondary eye positions has never been the subject of experimental study. Moreover, the implications of 3-D rotational eye kinematics for arm control have rarely even been considered. To judge by previous experiments (Wolpert et al., 1994; Henriques et al., 1998), correct prehensile compensation for visual distortion is hardly a foregone conclusion. Therefore, the current study had two goals: first, to quantify the degree of curvature in retinotopic projections following vertical eye rotations, and second, to determine how this is accounted for in the visuomotor transformation for pointing. The results demonstrate a specific nonlinear dependence of retinotopic projection on eye position that has been ignored by visual 
A
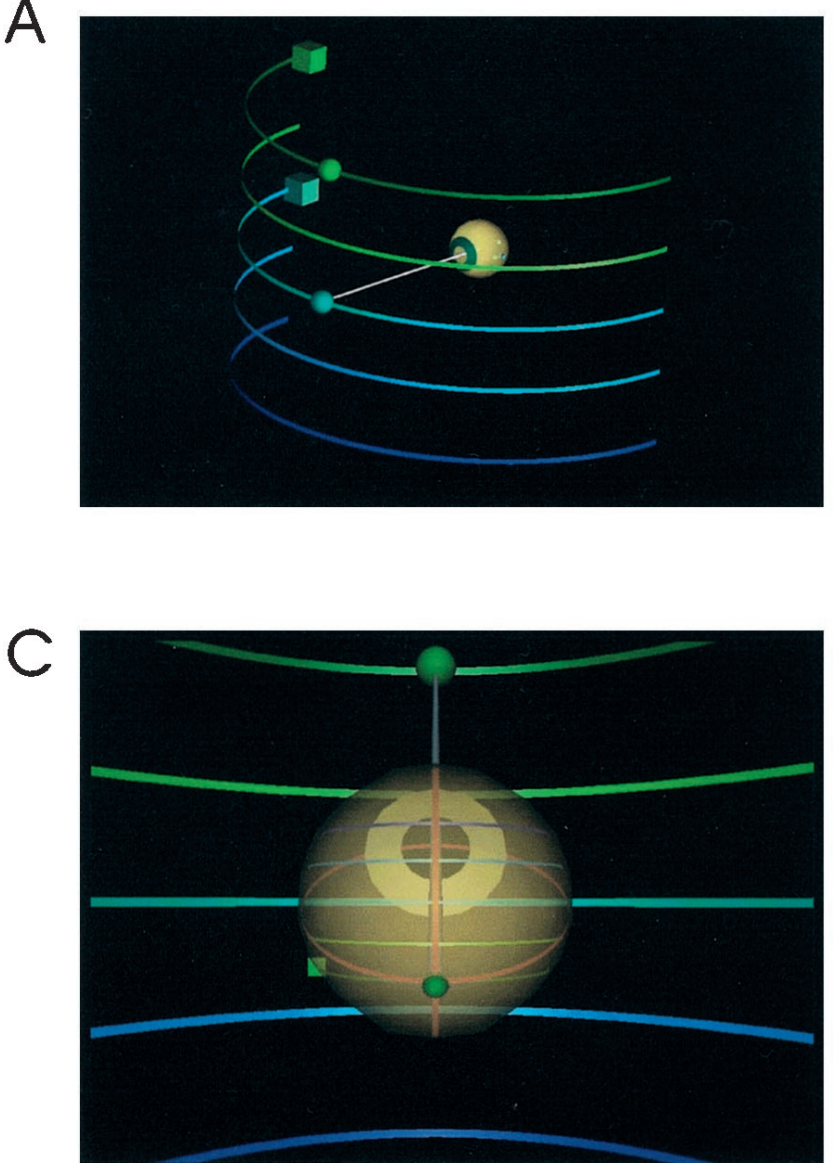

B

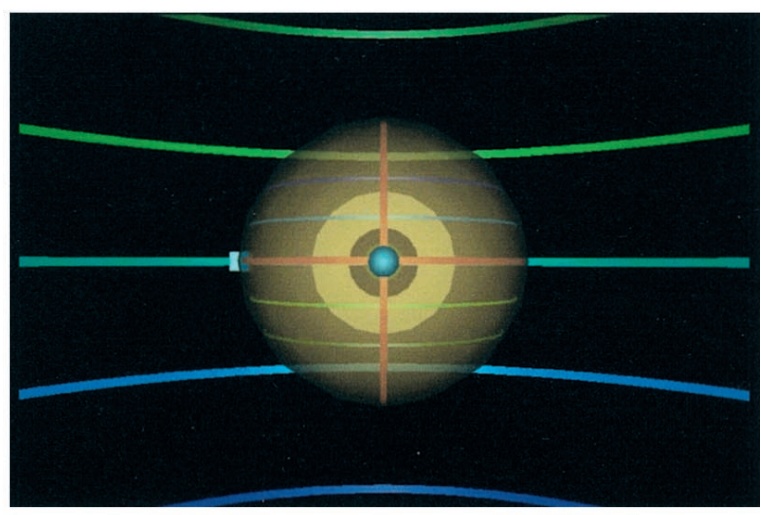

D

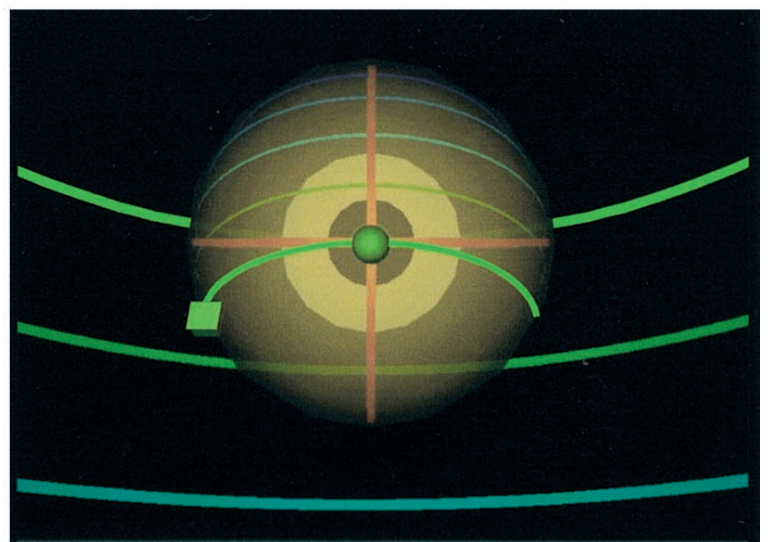

Figure 1. Simulated eye position-dependent geometry of retinal stimulation. A, Stimulus array viewed from a distance, showing the objective locations of its components. Simulated target pairs are located on five horizontal semicircles centered around the eye, elevated (in terms of gaze angle) at $30^{\circ}$ up, $15^{\circ}$ up, $0^{\circ}, 15^{\circ}$ down, and $30^{\circ}$ down. The green sphere and blue sphere indicate two possible fixation points, with two targets ( green and blue squares) placed $90^{\circ}$ to the right from the perspective of the eye (which currently points to center). $B$, Close up view of the semitransparent eye from behind while it looks toward the central blue sphere. The optically inverted projections of the stimulus lines onto the retina are visible. $C$, Similar view with the eye fixating the central target on the topmost line, as in $A$. D, Same situation as $C$ but now viewed from an eye-fixed perspective, looking down the line of gaze toward the top stimulus line. This simulation can be viewed as an interactive animation at http://www.physiology.uwo.ca/LLConsequencesWeb/index.htm.

neuroscience but not by the visuomotor transformations of the brain itself.

\section{THEORY}

To understand the geometry of retinal projection under vertical eye orientation, we simulated this geometry with the use of math described previously (Crawford and Guitton, 1997) and VRML, a virtual reality modeling language. Figure $1 A$ shows several simulated views of stimuli and eye orientations similar to those used in this study. The precise orientation and curvature of these particular lines were chosen for gaze angle invariance and motor invariance of the task described in Materials and Methods. Other patterns will be considered in Discussion. The main point to be taken here is that, even if these lines remain fixed in space, their curvature in retinal coordinates depends on eye position, as follows.

In terms of objective space (Fig. $1 A$ ), every point along each stimulus line is located at an equal angle of elevation from the center of the eye. Let us define the horizontal meridian of the eye to be the great circle described by the intersection of a horizontal plane through the eye at primary position (Fig. $1 B$, horizontal orange line). Thus, when the eye looks straight ahead, the whole central stimulus line, including the current fixation point $(\bigcirc)$ and a $90^{\circ}$ rightward target $(\square)$ project onto the horizontal retinal meridian as expected, and the projections of the other lines are (non-great circles) parallel to this, similar to lines of latitude. However, when the eye looks up (or down), the retinal projections of the stimuli curve vertically with respect to the eye-fixed horizontal meridian (Fig. $1 C, D$ ). From a space-fixed perspective $(C)$, the retinal projections of the stimulus lines remain horizontal, but the horizontal meridian of the eye (orange) looks curved. Conversely, from the perspective of the eye $(D)$ the horizontal retinal meridian is once again horizontal, but the retinal projections of the stimulus lines look curved. As a result, the retinal projection of a $90^{\circ}$ rightward target $(\square)$ falls on a point of the retinal projection line (highlighted) that is left and down relative to the central foveal region, signifying that the target is rightward and upward in retinal coordinates.

As a result, the sum of the vertical gaze angle and the retinal target vector would give a misestimate of actual target elevation in space, escalating in a nonlinear manner for points located progressively more peripherally along the line. Similar effects occurred for vertical lines and horizontal eye positions. In other words, even at pure secondary eye positions, target direction could only be computed through vector addition when the target 
and eye rotation are contained in the same one dimension. Left unaccounted for by some neural mechanism, this would result in mislocalizations of point targets (as well as eye positiondependent misperceptions of objective curvilinear shapes, as shown in Fig. 1).

The latter could be tested in a variety of perceptual and visuomotor systems. We chose pointing because (e.g., in contrast to eye movements) it normally correlates well with perceptual measures of vision (Gauthier et al., 1990; Wolpert et al., 1994). Moreover, the hand-arm system is clearly not organized in eye coordinates during straight-arm pointing but rather a body-fixed "Fick-like" coordinate system (Hore et al., 1992; Miller et al., 1992). Thus, for targets like those shown in Figure 1, an eccentric pointing movement from the central target $(\bigcirc)$ to the peripheral target $(\square)$ on a given horizontal line should show near-motor invariance at each level, with the arm essentially rotating about a body-fixed vertical axis. Therefore, there can be no confusion here about the coordinate frames of the visual input and motor output; a definite internal series of reference frame transformations from eye coordinates to body coordinates is required (Soechting and Flanders, 1989; Flanders et al., 1992; McIntyre et al., 1997; Henriques et al., 1998; Vetter et al., 1999). Furthermore, specific physiological processes in posterior parietal cortex and premotor-motor cortex have now been implicated in such eye-tohead-to-body transformations (Georgopoulos et al., 1982; Mushiake et al., 1997; Snyder et al., 1997). This is of some theoretical importance because, whereas previous models of noncommutative-rotational transformations have been applied to brainstem processes (Tweed and Vilis, 1987; Crawford and Guitton, 1997; Tweed et al., 1999), they can now potentially be applied to higher cortical functions.

\section{MATERIALS AND METHODS}

Experiments were performed under informed consent as approved by the York University Human Participants Review Subcommittee. Subjects were six right-handed humans (ages 23-45), with no previous knowledge of the experimental design and no known neuromuscular deficits. Each subject was seated in complete darkness with the head mechanically stabilized at the center of three 2-m-diameter Helmholtz coils. Orientations of the right eye and arm were measured using a 3-D search-coil technique (Hore et al., 1992; Henriques et al., 1998; Klier and Crawford, 1998). In brief, signals from a Skalar (Delft, The Netherlands) 3-D eye coil and a similar homemade 3-D coil secured to the upper arm were sampled at $50 \mathrm{~Hz}$ and converted off-line into eye-arm quaternions and pointing directions (Tweed et al., 1990).

The experimental procedure was designed to demonstrate the effect simulated in Figure 1 in the simplest possible way and to isolate its implications for arm movement. For the sake of simplicity, we only used pointing targets displaced horizontally from vertical gaze fixation points. To ensure that subjects could only reconstruct this stimulus pattern with the use of the visuomotor transformation under study, (1) the left eye was patched and no monocular depth cues were available during the experiment, and (2) subjects were not allowed uncontrolled exposure to this stimulus pattern. In other words, one could not tell where the targets were before the experiment began, and we did not provide subjects with visual feedback during the arm movement until the calibration procedures at the end of the experiment. (After experiments, some subjects reported anecdotally that targets did not indeed appear to be horizontally displaced but rather followed the retinocentric "fanning out" pattern illustrated in Fig. 1D). Finally, the full 3-D geometry of eye-arm coordination is highly complex, i.e., involving specific linkages between the centers of rotation of the eye, head, and arm segments (Flanders et al., 1992; Sabes and Jordan, 1997), but most of this geometry is not directly relevant to the question at hand. Therefore, we isolated the current effect by directly comparing the input-output relationships of retinal curvature (in eye coordinates) against body-centric errors in final arm angle (relative to appropriate controls).

During experiments, subjects were required to look and point toward
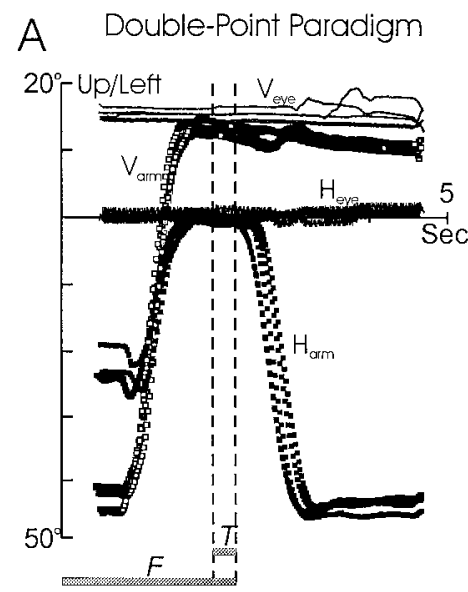

B Single-Point Paradigm
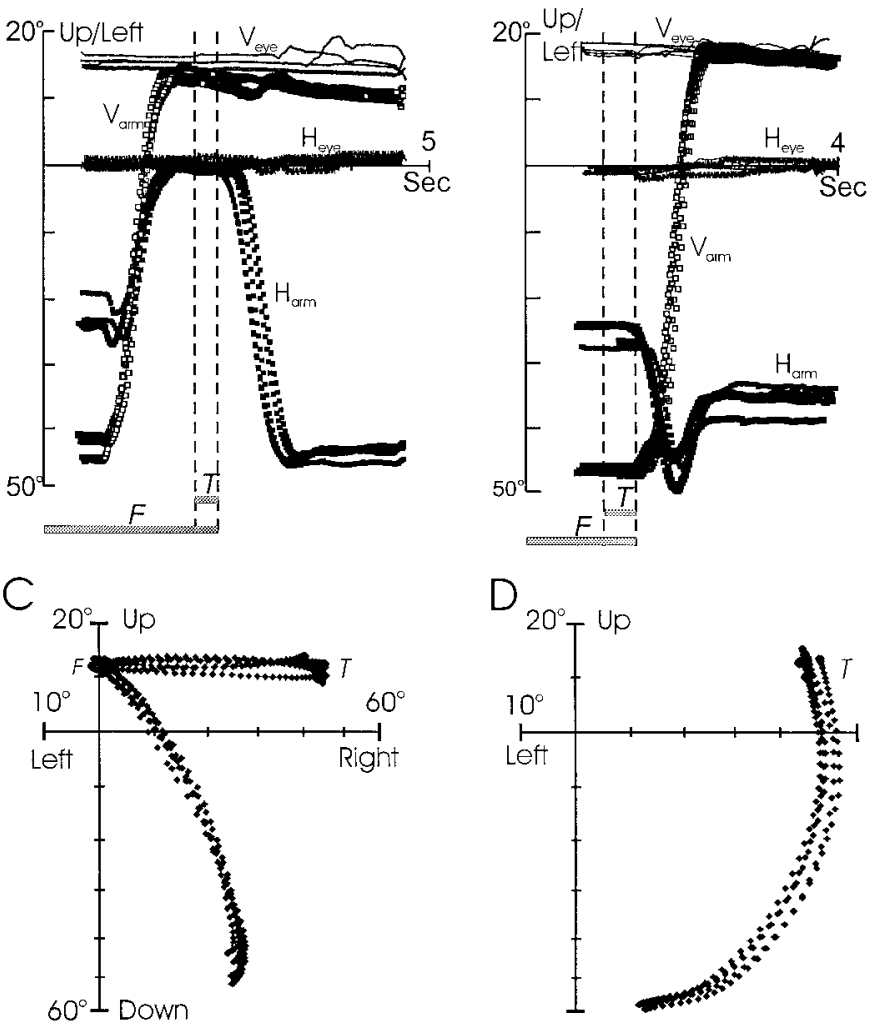

Figure 2. Experimental pointing paradigms. $A, B$, Examples of four eye and arm trajectories recorded during each of the two pointing paradigms, plotted as a function of time. $F$, Duration of fixation target. $T$, Duration of pointing target. In these particular cases, $F$ was $15^{\circ}$ up and $T$ was $60^{\circ}$ to its right. घ, Horizontal arm orientation. $\square$, Vertical arm orientation. Thick lines, Horizontal eye orientation. Thin lines, Vertical eye orientation. $A$, The double-point paradigm. Subject first pointed toward $F$ and then continued to fixate on $F$ while pointing toward $T$. $B$, The single-point paradigm. Subject pointed directly to $T$ while maintaining fixation on $F$. Subjects consistently showed a transient postmovement downward drift of the arm resembling saccadic pulse-step mismatch at all target levels in both paradigms. $C, D$, Corresponding 2-D trajectories of upper arm orientation for the same movements.

light-emitting diodes (LEDs), arranged in a pattern like that illustrated in Figure $1 A$. Specifically, LEDs were placed along five horizontal semicircles (positioned $30^{\circ}$ down, $15^{\circ}$ down, $0^{\circ}, 15^{\circ}$ up, and $30^{\circ}$ up), forming a vertical hemicylinder of $110 \mathrm{~cm}$ radius, centered on the right eye. As in Figure 1, the stimulus targets were arranged in horizontal pairs along these semicircles, with fixation targets $(\bigcirc)$ along the midline of each circle and pointing targets $(\square)$ located to the right (from the subject perspective). A total of 24 such fixation-pointing target pairs were used, placed in the configurations described below and illustrated in Results.

In each pointing trial, subjects began by monocularly fixating an illuminated LED fixation target $(F)$ located centrally along the vertical meridian, at which time a second target $(T)$ LED was briefly flashed to the right and at the same elevation (in space coordinates). Subjects were then required to maintain eye fixation while indicating the position of the rightward target with the use of one of two pointing paradigms (Fig. $2 A, B)$. This dissociation between gaze and pointing ensured that the arm did not simply follow the direction chosen by the gaze system, but it can also produce confounding errors related to nonhomogeneities in reading out the retinotopic map. However, our recent controls for this (Henriques et al., 1998; Henriques and Crawford, 2000) suggest that such errors are an order of magnitude smaller than the potential errors to be tested in the current study and mainly relate to misestimates in the magnitude of retinal displacement rather than its direction. For the purpose of analysis, final pointing direction was defined as the last stable arm orientation before the arm reaccelerated toward the next pointing or 

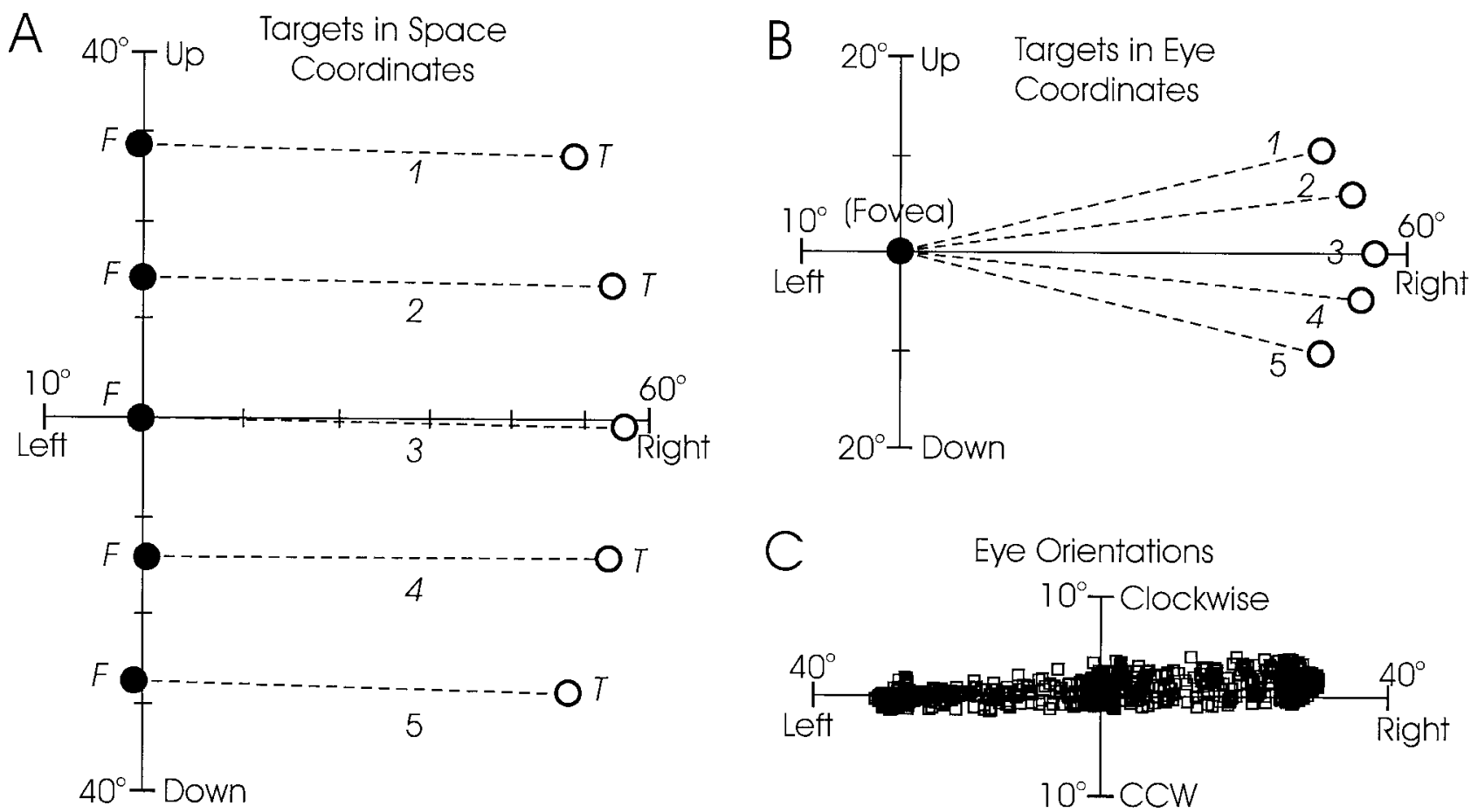

Figure 3. Stimulus locations in spatial and retinal frames in one typical subject. $A$, Target locations in space coordinates, computed from eye position signals recorded while subjects fixated each target. $\bullet$, Target location used for ocular fixation and initial pointing direction. $\bigcirc$, Target location used for final pointing direction. Dashed lines indicate the pairing of fixation and pointing targets during experiments. In this and subsequent figures, angular directions are represented with the use of unit-length vectors aligned with the pointing direction and projected onto a frontal plane (Klier and Crawford, 1998), such that the scale follows a sine function and the locations of oblique targets appear to be slightly distorted compared with their locations in translational space. $B$, Target directions $(\bigcirc)$ in retinal coordinates (right eye). The horizontal and vertical axes are the flat projections of the orange retinal meridian in Figure 1, $B$ and $D$, viewed from behind the eye, but the optical inversion is dispensed with so that rightward vectors indicate rightward targets, etc. To derive these vectors, the original direction vector for each rightward target $(O)$ in $A$ was rotated by the inverse of the average measured 3-D eye orientation vector while the subject fixated (๑) (Klier and Crawford, 1998). Thus, the fixation target (๑) now always corresponds to the fovea, and the horizontal coordinate axis corresponds to horizontal retinal meridian (defined here as the retinal arc intersected by the horizontal plane passing through the center of the eye when gaze is directed straight ahead). Note that the pattern of stimulation was symmetric about the horizontal meridian in this particular subject, whose Listing's plane of 3-D eye orientation vectors $(C)$ happened to align closely with the spatial frontal plane. However, in subjects with tilted Listing's planes (i.e., in which ocular torsion was a function of gaze angle), the pattern was predictably skewed either upward or downward, as described previously (Klier and Crawford, 1998). This is one reason why it is necessary to use 3-D eye orientation to compute retinocentric target vectors.

resting position. Finally, the resting position of the arm was held constant to minimize potentially confounding errors related to variations in initial arm position (Ghilardi et al., 1995; Vindras et al., 1998).

In the "double-point" paradigm (Fig. $2 A$ ) subjects were required to first point (arm fully extended) toward $F$ and then, only when both lights were extinguished, point toward the rightward target. An auditory tone signaled the subject to return the arm to a constant resting position. This paradigm resulted in saccade-like preprogrammed arm trajectories proceeding from $F$ to $T$ (Fig. $2 C$ ), which was useful for obtaining a graphic depiction of the results but was not ideal for quantification because these trajectories were time-consuming, tiring, and somewhat predictable. Therefore, this paradigm was only performed using the five standard horizontal $F-T$ target pairings illustrated in Results (Fig. 3A).

In contrast, in the "single-point" paradigm (Fig. $2 B$ ), the subject did not point at $F$ but rather pointed directly from the resting position toward $T$ after it had flashed. With targets presented in random order, this paradigm gave rise to otherwise unpredictable arm trajectories (in contrast to the double-point paradigm). Moreover, because this paradigm required less time and was much less tiring, it was applied to a much larger array of horizontal $F-T$ target pairings (24 pairs in total). Specifically, the spatial coordinates of these pairs were: five midline $F$ targets ( $30^{\circ}$ down, $15^{\circ}$ down, $0^{\circ}, 15^{\circ}$ up, $30^{\circ}$ up), each paired with one $T 60^{\circ}$ to its right, and one $T 80^{\circ}$ to its right; two $F$ targets at $10^{\circ}$ right $\left(30^{\circ}\right.$ up and $30^{\circ}$ down), each paired with one $T 50^{\circ}$ to its right and one $T 70^{\circ}$ to its right; and five targets at $20^{\circ}$ right $\left(30^{\circ}\right.$ down, $15^{\circ}$ down, $0^{\circ}, 15^{\circ}$ up, $30^{\circ}$ up $)$, each paired with one $T 40^{\circ}$ to its right and one $T 60^{\circ}$ to its right. These pairings were selected on the basis of simulations to give a broad, even distribution of predicted errors to test against the actual results. This data provided the primary quantitative test in our experiment.
After placing the Skalar contact lens in the subject's eye, the subject performed the preceding paradigms in the following order. First, subjects did the single-point paradigm (Fig. 2B), performing five repetitions for each of the 24 target pairs (see Fig. 5), in random order. Second, they did the double-point paradigm (Fig. $2 A$ ), performing a total of 10 repetitions for each of five standard target pairs (Fig. $3 A$ ). These pairs were performed in counterbalanced order (top-to-bottom-to-top, etc.) so that any resulting fatigue would have no systematic influence on the results. Third, they did the controls for the double-point paradigm. These were the same as the double-point paradigm, but pointing was done with both the arm and target visible (in dim light) for full visual feedback. Fourth, they did the controls for the single-point paradigm. These were the same as the single-point paradigm, but pointing was done with both the arm and target visible for an extended period of time $(3 \mathrm{sec})$, allowing time for corrective movements with full visual feedback.

Several additional controls were then performed. Reference eye and arm positions were recorded while subjects pointed toward the central target with full visual feedback and then with the arm aligned straight ahead (for quantitative analysis of arm rotation in shoulder coordinates). Then, each of the targets ( $T$ ) were sequentially illuminated for $2 \mathrm{sec}$, and subjects were instructed to stare at each one in turn. Eye coil signals at the center of each of these fixation "centroids" were later selected for conversion into unit vectors pointing toward the target (Tweed et al., 1990), which were used to express target direction in space coordinates (Klier and Crawford, 1998). These data were later used to compute target directions in retinal coordinates (Fig. 3) and hence predicted arm trajectories based on a linear model (Fig. 4). Coil signals were used (as opposed to objective geometric measurements) so that any small errors caused by magnetic field nonhomogeneities would be common to both 

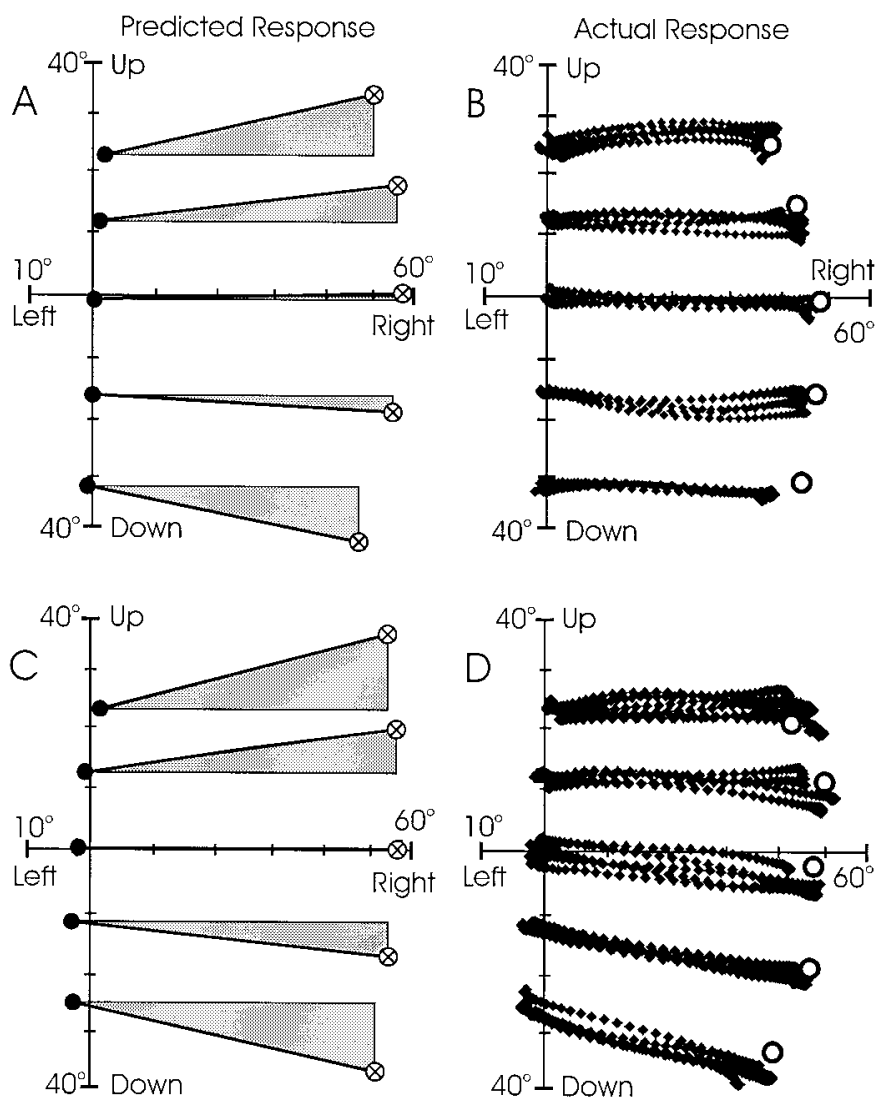

Figure 4. Predicted and actual pointing trajectories from the doublepoint paradigm in two subjects. $A$, Predicted responses. $\boldsymbol{\bullet}$, Average initial 2-D arm position. $\otimes$, Final positions predicted by traditional models that compute the target direction based on addition of the current gaze direction with the retinal vector (Hallet and Lightstone, 1976; Zee et al., 1976; Mays and Sparks, 1980; Howard, 1982; Zipser and Andersen, 1988; Flanders et al., 1992; Miller, 1996; Bockisch and Miller, 1999) or in this task if the arm were simply displaced in the direction coded by the retina. Gray wedges, Predicted angle of error from the (due rightward) ideal trajectory. $B$, Actual responses. Corresponding actual angular arm trajectories ( $)$ for five movements at each height, done in complete darkness to a previously flashed target. $\bigcirc$, Control pointing directions with full visual feedback of arm and target. $C, D$, Similar data for the subject whose arm trajectories came closest to following the predictions of a linear model. Note that the predicted pattern of error for each subject was not generally identical because it was also influenced by the orientation of Listing's plane, which varies between subjects (Klier and Crawford, 1998). Also note that the arm angles were slightly different than those of the eye (Fig. $3 A$ ) because they do not share the same center of rotation, but otherwise this task provides motor invariance at different vertical levels in terms of the axis of arm rotation during pointing (Hore et al., 1992; Miller et al., 1992).

the predicted and actual arm movement data. We also measured random saccades between nine targets in a $\pm 30^{\circ}$ horizontal-vertical grid (illuminated $1 \mathrm{sec}$ each in dark), to establish the orientation of Listing's plane. This was not necessary to compute the following results but was useful for interpretation of certain second-order modulations on the main effect (Klier and Crawford, 1998). Finally, eye coil calibration procedures were followed (Henriques et al., 1998; Klier and Crawford, 1998).

\section{RESULTS}

Figure $3 A$ illustrates the stimulus positions used for the doublepoint paradigm as measured in a space-fixed coordinate system centered on the right eye in which the targets formed horizontal pairs $(\bullet, \bigcirc)$. Figure $3 B$ then illustrates the computed directions of the rightward targets $(\bigcirc)$ in an eye-fixed coordinate system, computed by rotating the rightward positions from $A$ by the inverse of 3-D eye orientation at the leftward fixation point (O) (Klier and Crawford, 1998). This procedure gives the positions of the targets as they would appear to the right retina when subjects fixated the leftward target of each pair. The main point of Figure 3 is that targets $(\bigcirc)$ that were displaced purely horizontally from fixation points $(\mathbf{O})$ in space coordinates (Fig. $3 A$ ) were displaced in different oblique directions relative to the fovea in oculocentric coordinates (Fig. 3B), tilting as a function of initial eye position. This confirmed the prediction of our simulation (Fig. 1) in which target images fell on lines of projection that curved with respect to the horizontal retinal meridian as a function of eye orientation (Fig. 1). How then would subjects perform when required to point and fixate toward each of the midline targets (O) and then maintain fixation while pointing toward the briefly flashed rightward target $(\bigcirc)$ in the complete absence of visual feedback?

Figure 4 shows the results of this test in two subjects (top row vs bottom row). $A$ and $C$ show the average initial pointing directions (-) to the midline fixation lights, plotted in a space-fixed coordinate system centered about the shoulder. These arm angles are not identical to the eye-centered target angles in Figure 3 because the arm and eye do not share the same center of rotation, and the arm generally points to align the finger with the line of gaze rather than pointing directly at the target (Flanders et al., 1992). Nevertheless, as confirmed by our control measures of arm kinematics, the task required these subjects to rotate the arm directly to the right. However, if the visuomotor transformation simply generated an arm displacement command in the direction of the retinal target vector (computed as in Fig. $3 B$ ) or added these vectors to the current gaze direction vector to compute target direction, it would produce the fanning out pattern of pointing responses $(\otimes)$ illustrated in Figure 4, $A$ and $C$. Note that the resulting position-dependent pattern of errors (depicted by the gray wedges) would arise from a failure to compensate for the nonlinear eye position-dependent pattern of raw retinal signals such as those shown in Figure $3 B$.

Figure $4 B$ shows the actual arm trajectories $(\diamond)$ of a subject that showed near-ideal trajectories for this task. Like the data of most of our subjects, these arm trajectories showed little or no tendency to fan out as a function of initial vertical eye position, pointing very accurately relative to visually guided controls $(\bigcirc)$. Only one subject showed a partial tendency to follow the fanning out pattern, as seen in Figure $4 D$. In either case, final pointing directions showed little systematic variation across counterbalanced trials, although the variance was slightly higher for the more eccentric targets (SDs between trials, averaged across subjects, were $2.37^{\circ}, 2.17^{\circ}, 2.04^{\circ}, 2.29^{\circ}$, and $2.69^{\circ}$ top $F-T$ pair to bottom, respectively). This suggests that the visuomotor transformation was consistent and that subjects were not unduly influenced by fatigue. Averaged across subjects, the slope of the actual vertical errors in pointing relative to controls (Fig. $4 B, D$ ) as a function of predicted errors (Fig. $4 A, D$ ) across target pairs was only $-0.26 \pm 0.55$ (SD). This negative slope signifies a slight overcompensation for eye position. In summary, the double-point test suggested that our subjects compensated for the eye position dependence of their retinal signals, sometimes partially, sometimes completely, and sometimes a bit too much. However, considering that the slopes fit to these data were based on only five $F-T$ target pairings, this measure was not as reliable as that shown in the next test.

To confirm these observations quantitatively across a much 

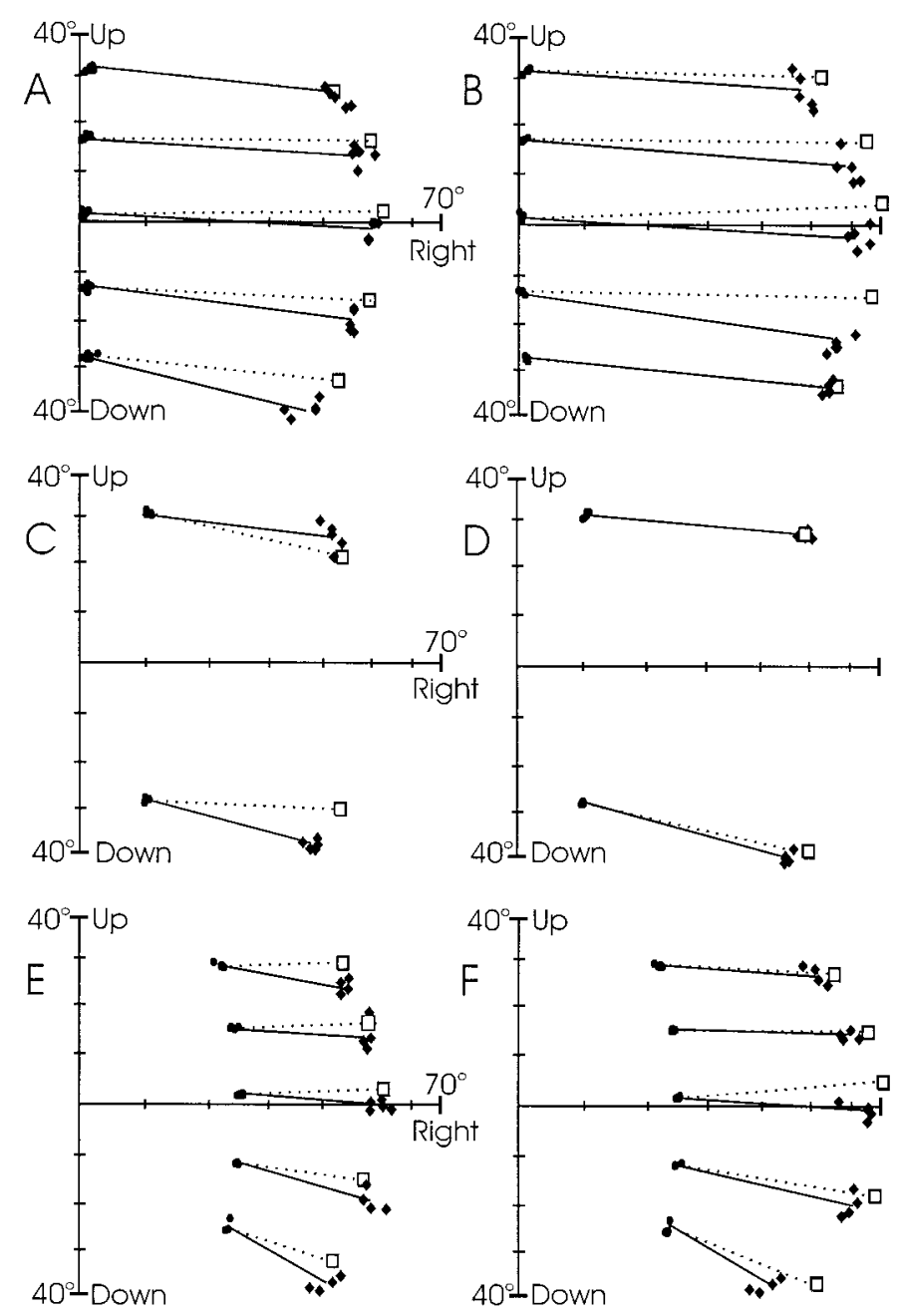

Figure 5. Performance of one typical subject in the single-point paradigm. - Measured fixation directions. $\square$, Desired pointing direction to $T$ determined from controls with full visual feedback. $\bullet$, Actual final pointing directions in the absence of visual feedback. Horizontal lines connect corresponding fixation and pointing data for illustrative purposes only; they do not represent trajectories or any other meaningful variable. Data are arranged $(A-F)$ according to the horizontal locations of the fixation and pointing targets for clarity, but targets were randomized during the experiment.

larger data range, we compared the errors predicted by the vector addition model with actual errors measured relative to control values. This was done using data from the single-point paradigm (Fig. 2B), which, for reasons explained in Materials and Methods, allowed us to explore the broader range of $F-T$ target pairings shown in Figure 5. As mentioned above, these particular target pairings were chosen so as to provide an even distribution of predicted pointing errors (based on simulations). Furthermore, because this paradigm involved unpredictable arm trajectories in various directions toward randomly presented targets, it further controlled for the possibility that subjects might have settled into a rote or cognitively guided pattern of horizontal trajectories in the double-point paradigm.

Each panel in Figure 5 shows ocular fixation directions ( ) for one subject, joined (for purpose of display) to corresponding control pointing directions $(\square)$. The latter were recorded at the end of the experiment while the subject pointed toward the rightward targets with full visual feedback of both the arm and
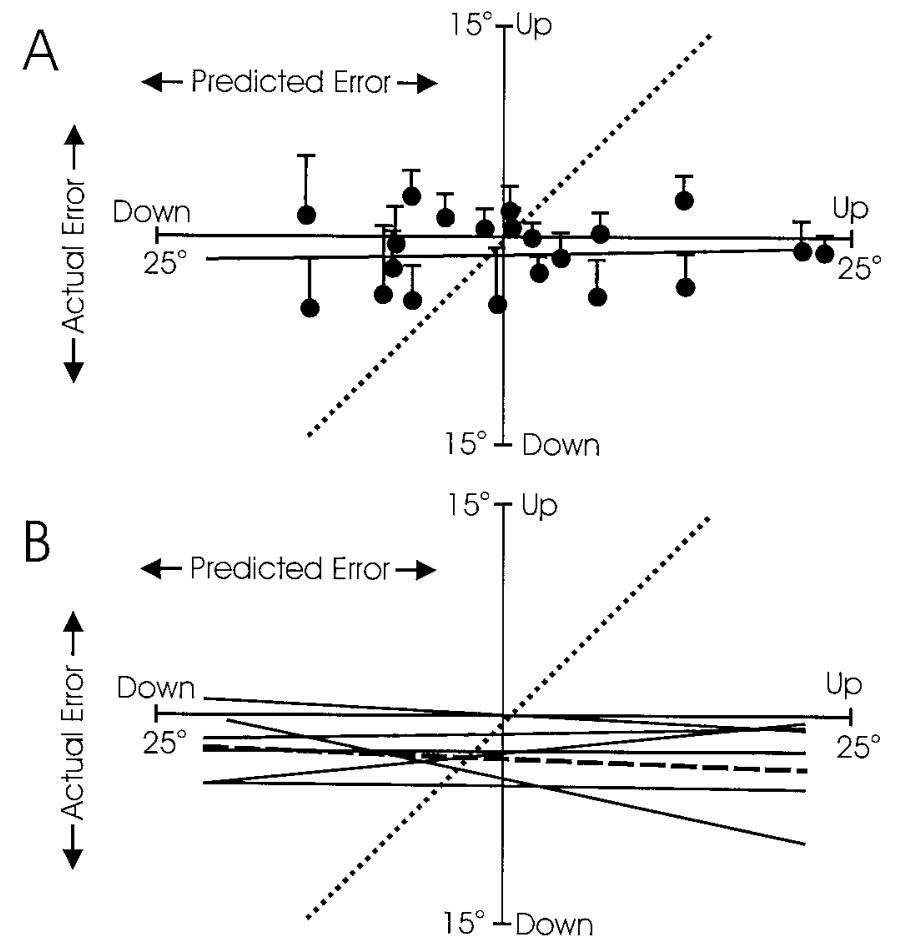

Figure 6. Summary of actual (vertical axis) versus predicted (horizontal axis) pointing errors in the single-point paradigm. $A$, Individual data points for each of 24 stimulus pairs, each averaged across five movements, for one typical subject. The average vertical component of predicted angular error (computed from initial 3-D fixation positions and target vector measurements) is plotted along the horizontal axis. This signifies the constant error that would be made if the system failed to account for the measured vertical curvatures in retinal location induced as a function of initial eye orientation. Average angular errors in the actual responses (relative to ideal responses with visual feedback) are plotted along the vertical axis. Vertical error bars show SD across pointing trials for each target (horizontal variance was too small for graphic display). Also shown is a line fit by regression to the average points. $B$, Solid lines, Similar lines of regression fit for all six subjects. Hatched lines, Alignment of the data parallel to the horizontal axis represents complete rotational compensation for eye orientation, whereas alignment of the data parallel to the slope of unity (dotted lines) represents zero rotational compensation for eye orientation.

target. Note that the final pointing directions $(\bullet)$ followed arm trajectories (data not shown) with variable horizontal and vertical components from the initial resting position in this single-point paradigm. Final pointing responses $(\bullet)$ consistently undershot the control values $(\square)$ vertically, perhaps because of pulse-step mismatch (Fig. 2B) or a consistent misperception of initial arm position (Ghilardi et al., 1995; Vindras et al., 1998). However, the main point of the figure is that there was little or no systematic eye position-dependent deviation in pointing responses across the entire pattern of $F-T$ target pairs.

To quantify the latter observation, we computed the average vertical pointing error, relative to controls, for each of the 24 target pairings in the single-point paradigm, and plotted this as a function of the vertical component of retinal target vector (computed for each $F-T$ target pairing as shown in Fig. 3). The latter corresponds to the error that subjects would make if they failed to account for the effect of vertical eye position on retinal curvature when computing target location. Figure $6 A$ shows such a plot for one typical subject. Figure $6 B$ then shows regression lines fit to similar data from all 6 subjects. A slope of one (dotted lines) would 
suggest that no correction for the position effect had occurred, whereas a slope of zero would suggest perfect correction. Although individuals showed a consistent vertical offset unrelated to eye position (Henriques et al., 1998) and various stochastic errors (Henriques and Crawford, 2000), they showed little or no systematic error as a function of eye position. In fact, only the subject with the largest slope (at -0.209) showed a slope significantly different from zero. The average slope across subjects (hatched lines) was $-0.019 \pm 0.095(\mathrm{SD})$, suggesting that the visuomotor transformation for pointing had made the correct compensation.

\section{DISCUSSION}

It has long been known that the brain must account for the vertical and horizontal angles of gaze direction to correctly localize visual stimuli, as well as the torsional angle of eye orientation about the gaze axis (von Helmholtz, 1867; Hallet and Lightstone, 1976; Zee et al., 1976; Mays and Sparks, 1980; Howard, 1982; Mittelstaedt, 1983; Zipser and Andersen, 1988; Haustein and Mittelstaedt, 1990; Flanders et al., 1992; Miller, 1996; Wade and Curthoys, 1997; Bockisch and Miller, 1999). Moreover, we have recently shown that the brainstem saccade generator must compensate for 3-D eye orientations to generate accurate saccades from tertiary eye positions (Klier and Crawford, 1998). However, the current study is the first to experimentally demonstrate that simple rotation of the eye to an upward or downward orientation produces complex curvatures in the correspondence between horizontal lines in visual space and their retinal projections. Furthermore, we have shown that the visuomotor control system for pointing compensates for these eye position-dependent effects. This is noteworthy because, based on the known neurophysiology of frontal (Georgopoulos et al., 1982; Mushiake et al., 1997) and parietal (Snyder et al., 1997) cortex, any such transformation would have to occur at a cortical level before primary motor cortex, most likely within prefrontal (Mushiake et al., 1997) or posterior parietal cortex (Snyder et al., 1997).

Clearly, the locations of visual targets in 3-D space will influence the pattern of retinal stimulation and the predicted motor responses to those stimuli. The particular semicircular pattern of horizontal stimulus lines that we used, giving rise to retinal projections resembling lines of latitude (Fig. $1 B$ ), were specifically selected to give horizontal arm trajectories in our double-point paradigm (Fig. 4B). However, how well does this effect generalize? Figure 7 shows that if our stimulus array were replaced by a series of horizontal Euclidean lines at different vertical levels on a fronto-parallel plane (Fig. $7 A$ ), it would now give rise to retinal projections resembling lines of longitude (except horizontally arranged) (Fig. 7B). With this unique arrangement, the current line of regard would stimulate the horizontal retinal meridian independent of vertical gaze angle (Fig. $7 C$ ), but this simply reverses the problem for visuomotor transformations; just as a nonhorizontal retinal code at upward gaze mapped onto horizontal arm displacements in our experiment (Fig. 4B), a horizontal displacement in retinal coordinates (Fig. 7D) would now require a nonhorizontal, oblique displacement of the arm. Moreover, there is obviously nothing special about vertical eye orientations and horizontal lines; the same geometry would hold for any large linear component of a stimulus that is orthogonal to the displacement of eye orientation from center.

Because this basic ocular geometry will affect most aspects of spatial vision and every visuomotor transformation (Klier and Crawford, 1998), each such system would require a compensatory
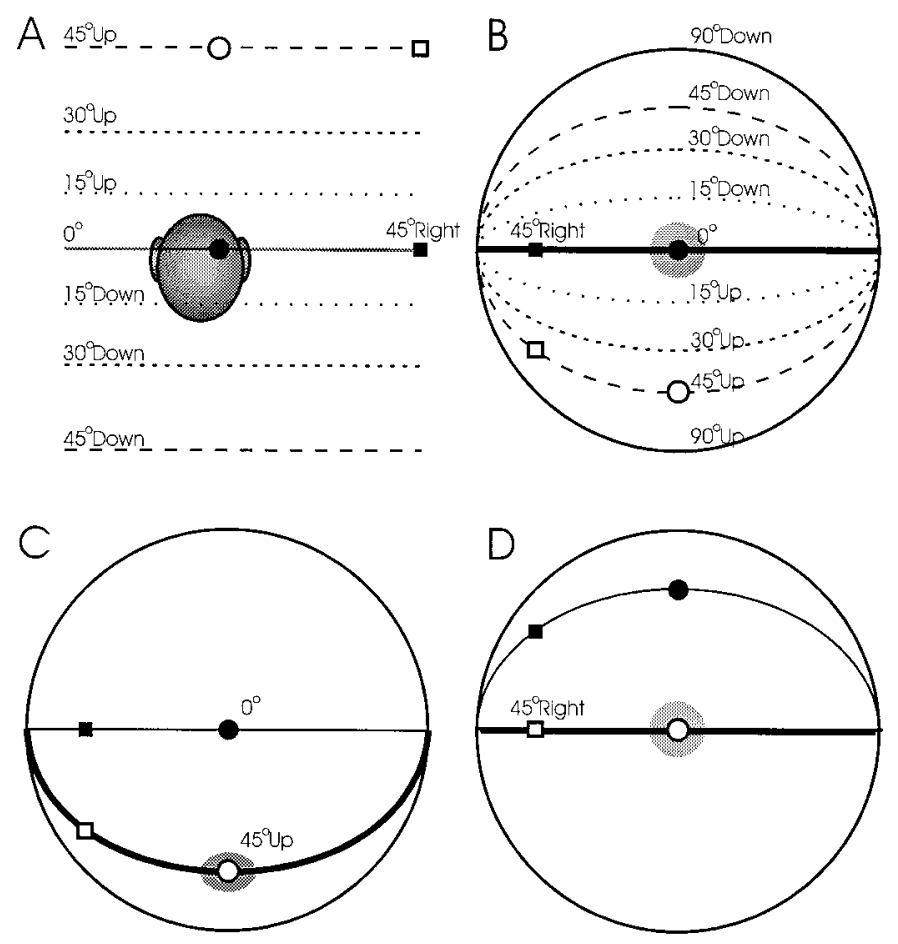

Figure 7. Retinal projection geometry of straight (in the Euclidean sense) horizontal lines in a fronto-parallel plane. $A$, Lines viewed from behind a semi-transparent "head" indicating subject's position. A horizontal pair of targets is placed straight ahead $(\mathbf{O})$ and $45^{\circ}$ right $(\square)$, with a similar pair $(\bigcirc, \square)$ at $45^{\circ}$ angle up (gaze angle). $B$, Projections of lines and targets (same symbols) onto retina, as viewed from behind. Gray disk, Foveal region. Thick line, Eye-fixed great circle through the fovea that defines the horizontal retinal meridian. Note that the retinal projections of the Euclidean lines resemble nonparallel lines of longitude (except horizontally arranged). $C$, Projections of the same targets (minus irrelevant lines) onto the retina, viewed from the same space-fixed perspective but now with gaze rotated $45^{\circ}$ upward so that the upper target (ם) stimulates the fovea (which, being at the back of the eye, is rotated down). Note that the current line of regard again falls on the horizontal retinal meridian. $D$, Same retinal projection pattern as $C$ but viewed from an eye-fixed perspective, along the visual axis. Note that the target $(\square)$ that was up and right in space coordinates now stimulates a retinal point signifying purely rightward displacement in retinal coordinates. However, for the arm to point accurately from $\bigcirc$ to $\square$ in our double-point paradigm, it would have to follow an oblique rightward-downward trajectory, again requiring a multiplicative reference frame transformation.

neural mechanism, either implemented at a global level (Bockisch and Miller, 1999) or in parallel for each separate spatial-motor system (Snyder et al., 1997). Those who favor the global representation hypothesis might argue that the brain may normally use visual depth information to reconstruct the curvatures of lines in space such as those used in our study. However, depth information was not available in the current study, forcing subjects to rely on the only possible available information: monocular retinal information and any available internal representation of 3-D eye orientation. This shows that the system has this information and the capacity to use it to reconstruct angular target direction and thus probably does make use of this capability in real life. Furthermore, 3-D eye orientation varies with vergence angle (Mok et al., 1992; Van Rijn and Van den Berg, 1993), and thus accurate binocular depth information also requires some internal knowledge or assumptions of 3-D eye position (Tweed, 1997; Backus et al., 1999).

How then does the cerebral cortex perform such eye position 
compensations for this and the other previously known eye position dependencies? Based on previous traditions in modeling such transformations (Haustein and Mittelstaedt, 1990), one might conclude that the brain requires a (growing) array of special purpose mechanisms to account for each of these seemingly separate effects. Such corrective mechanisms might be implemented physiologically in a side loop, such as the cerebellum. However, a much more parsimonious model is possible. For example, to compute target direction in retinal coordinates, we rotated the objective target vector by the inverse of 3-D eye orientation (in contrast to the translation-based vector algebra used in most oculomotor studies). Conversely, the brain could construct a head-centric representation of angular target direction by rotating the retinal target vector by an internal representation of 3-D eye orientation. As demonstrated in our previous theoretical oculomotor investigation, this operation will work for any and all retinal target vectors and eye orientations (Crawford and Guitton, 1997), potentially even those encountered in pathological eye movements. Such rotatory processes are not additive but rather multiplicative, with different implications for the organization of realistic neural networks (Smith and Crawford, 1998; Tweed et al., 1999), including specific types of cross talk (Crawford and Guitton, 1997) between dissimilar components of retinal signals and eye position signals.

Two possible arguments against this algorithm might be raised. First, it would be very computationally demanding if performed on input from every point in visual space. However, we have argued elsewhere that such transformations need only apply to targets that have been specifically selected for action or cognition (Henriques et al., 1998; Klier and Crawford, 1998). Second, the preceding scheme would seem to suggest extensive head-centric maps of visual space that are not predominant in actual brain physiology (Georgopoulos et al., 1982; Duhamel et al., 1992; Moschovakis and Highstein, 1994; Mushiake et al., 1997; Snyder et al., 1997; Colby and Goldberg, 1999). However, we have recently confirmed that such multiplicative reference frame transformations can be entirely implicit within a neural net without ever developing an explicit representation of target position in space (Smith and Crawford, 1998). In other words, a cortical retinotopic vector can be converted into a head-centric or bodycentric displacement command simply by modulating it as a function of eye or head position. These theoretical arguments are consistent with the general physiology of visuomotor cortex (Georgopoulos et al., 1982; Moschovakis and Highstein, 1994; Maunsell, 1995; Mushiake et al., 1997; Colby and Goldberg, 1999) and are particularly relevant for the recently observed gazecentered but eye position-dependent behavior in the parietal reach region (Snyder et al., 1997; Andersen et al., 1998; Batista et al., 1999).

If perceptual and visuomotor systems do indeed use parallel, independent streams within the cerebral cortex (Milner and Goodale, 1995; Andersen et al., 1998; Bockisch and Miller, 1999), it would be a mistake to conclude that the current experiment shows that all of these systems account for the effects of eye orientation on retinal geometry. However, if initial information is stored in retinal coordinates, as we have suggested above, it is reasonable to hypothesize that visuomotor transformations downstream would use the correct motor transformation whenever feedforward accuracy is important in their behavioral task. Moreover, cognitive systems would also have to account for the changes of relative curvature in lines as a function of gaze angle to perceive allocentric visual space as constant.
Note that curvature is a relative term, always defined relative to some default notion of straightness. Considering our common sense notions of Euclidean straightness, it seems that higher level systems would probably use the projection pattern illustrated in Figure 7 as a default measure. (This is consistent with anecdotal reports from our subjects that our stimulus array did not form parallel lines). Again, having such a reference measure does not rid visual perception of the nonlinear geometric problems related to eye position. For example, consider the perception of moving objects, as in vertical ocular pursuit of an upward-translating horizontal object. Retinal geometry requires that the retinal projections of nonlinear stimuli would curve with respect to fixed retinal landmarks as it moves, as a function of current eye position (Fig. 1). For the shape and rigidity of such objects to be correctly judged (without previous knowledge), raw retinal representations would have to be rotated by current eye orientation at each point in time.

Such theoretical observations advocate the importance of the subtle eye position-dependent visual responses reported in such diverse areas as occipital cortex (Galletti and Battaglini, 1989; Trotter and Celebrini, 1999), posterior parietal cortex (Zipser and Andersen, 1988; Andersen et al., 1998; Batista et al., 1999), and frontal cortex (Boussaoud et al., 1993). However, the current study suggests that previous analyses of cortical visuomotor responses based solely on the theoretical framework of vector addition may have not yet revealed the full extent of their physiological capacity.

\section{REFERENCES}

Andersen RA, Snyder LH, Batista AP, Buneo CA, Cohen YE (1998) Posterior parietal areas specialized for eye movements (LIP) and reach (PRR) using a common coordinate frame. Novartis Found Symp 218:109-122.

Backus BT, Banks MS, van Ee R, Crowell JA (1999) Horizontal and vertical disparity, eye position, and stereoscopic slant perception. Vision Res 39:1143-1170.

Batista AP, Buneo CA, Snyder LH, Andersen RA (1999) Reach plans in eye-centred coordinates. Science 285:257-260.

Bockisch CJ, Miller JM (1999) Different motor systems use similar damped extraretinal eye position information. Vision Res 39:1025-1038.

Boussaoud D, Barth TM, Wise SP (1993) Effect of gaze on apparent visual responses of monkey frontal cortex neurons. Exp Brain Res 91:202-212.

Colby C, Goldberg G (1999) Space and attention in parietal cortex. Annu Rev Neurosci 22:319-350.

Crawford JD, Guitton D (1997) Visuomotor transformations required for accurate and kinematically correct saccades. J Neurophysiol 78:1447-1467.

Duhamel J-R, Colby CL, Goldberg ME (1992) The updating of the representation of visual space in parietal cortex by intended eye movements. Science 255:90-92.

Flanders M, Helms Tillery SI, Soechting JF (1992) Early stages in a sensorimotor transformation. Behav Brain Sci 15:309-362.

Galletti C, Battaglini PP (1989) Gaze-dependent visual neurons in area V3A of monkey prestriate cortex. J Neurosci 9:1112-1125.

Gauthier GM, Nommay D, Vercher J-L (1990) The role of ocular muscle proprioception in visual localization. Science 249:58-61.

Georgopoulos AP, Kalaska JF, Caminiti R, Massey JT (1982) On the relations between the direction of two-dimensional arm movements and cell discharge in primate motor cortex. J Neurosci 2:1527-1537.

Ghilardi MF, Gordon J, Ghez C (1995) Learning a visuomotor transformation in a local area of work space produces direction biases in other areas. J Neurophysiol 73:2535-2539.

Hallet PE, Lightstone AD (1976) Saccadic eye movements to flashed targets. Vision Res 16:107-114.

Haustein W, Mittelstaedt H (1990) Evaluation of retinal orientation and gaze direction in the perception of the vertical. Vision Res 30:255-262.

Henriques DYP, Crawford JD (2000) Direction-dependent distortions 
of retinocentric space in the visuomotor transformation for pointing. Exp Brain Res, in press.

Henriques DYP, Klier EM, Smith MA, Lowy D, Crawford JD (1998) Gaze-centered remapping of remembered visual space in an open-loop pointing task. J Neurosci 18:1583-1594.

Hore J, Watts S, Vilis T (1992) Constraints on arm position when pointing in three dimensions: Donders' law and the Fick gimbal strategy. J Neurophysiol 68:374-383.

Howard IP (1982) Human visual orientation. New York: Wiley.

Klier EM, Crawford JD (1998) The human oculomotor system accounts for 3-D eye orientation in the visual-motor transformation for saccades. J Neurophysiol 80:2274-2294.

Liu L, Schor CM (1998) Functional division of the retina and binocular correspondence. J Opt Soc Am A Opt Image Sci Vis 15:1740-1755.

Maunsell JH (1995) The brain's visual world: representation of visual targets in cerebral cortex. Science 270:764-769.

Mays LE, Sparks DL (1980) Saccades are spatially, not retinotopically coded. Science 208:1163-1164.

McIntyre J, Stratta F, Lacquantiti F (1997) Viewer-centered frame of reference for pointing to memorized targets in three-dimensional space. J Neurophysiol 78:1601-1618.

Miller JM (1996) Egocentric localization of a perisaccadic flash by manual pointing. Vision Res 36:837-851.

Miller LE, Theeuwen M, Gielen CCAM (1992) The control of arm pointing movements in three dimensions. Exp Brain Res 90:415-426.

Milner AD, Goodale MA (1995) The visual brain in action. Oxford: Oxford UP.

Mittelstaedt H (1983) A new solution to the problem of the subjective vertical. Naturwissenschaften 70:272-281.

Mok D, Ro A, Crawford JD, Vilis T (1992) Rotation of Listing's plane during vergence. Vision Res 32:2055-2064.

Moschovakis AK, Highstein SM (1994) The anatomy and physiology of primate neurons that control rapid eye movements. Annu Rev Neurosci 17:465-488.

Mushiake H, Tanatsugu Y, Tanji J (1997) Neuronal activity in the ventral part of premotor cortex during target-reach movement is modulated by direction of gaze. J Neurophysiol 78:567-571.

Sabes PN, Jordan MI (1997) Obstacle avoidance and a perturbation sensitivity model for motor planning, J Neurosci 17:7119-7128.
Smith MA, Crawford JD (1998) Accounting for 3-D eye rotations in the mechanisms for spatial memory and visuomotor transformation. Soc Neurosci Abstr 24:1742.

Snyder LH, Batista AP, Andersen RA (1997) Coding of intention in the posterior parietal cortex. Nature 386:167-170.

Soechting JF, Flanders M (1989) Errors in pointing are due to approximations in sensori-motor transformations. J Neurophysiol 62:595-608.

Trotter Y, Celebrini S (1999) Gaze direction controls response gain in primary visual-cortex neurons. Nature 398:239-342.

Tweed D (1997) Visual-motor optimization in binocular control. Vision Res 14:1939-1951.

Tweed D, Vilis T (1987) Implications of rotational kinematics for the oculomotor system in three dimensions. J Neurophysiol 58:832-849.

Tweed D, Cadera W, Vilis T (1990) Computing three dimensional eye positions queternions and eye velocity from search coil signals. Vision Res 30:97-110.

Tweed DB, Haslwanter TP, Happe V, Fetter M (1999) Noncommutativity in the brain. Nature 399:261-263.

Van Rijn LJ, Van den Berg AV (1993) Binocular eye orientation during fixations: Listing's law extended to include eye vergence. Vision Res 33:691-708.

Vetter P, Goodbody SJ, Wolpert DM (1999) Evidence for an eyecentered spherical representation of the visuomotor map. J Neurophysiol 81:935-939.

Vindras P, Desmurget M, Prablanc C, Viviani P (1998) Pointing errors reflect biases in the perception of the initial hand position. J Neurophysiol 79:3290-3294.

von Helmholtz H (1867) Handbuch der Physiologischen Optik. Hamburg, Germany: Voss.

Wade SW, Curthoys IS (1997) The effect of ocular torsional position on perception of the roll-tilt of visual stimuli. Vision Res 37:1071-1078.

Wolpert DM, Ghahramani Z, Jordan MI (1994) Perceptual distortion contributes to the curvature of human reaching movements. Exp Brain Res 98:153-156.

Zee DS, Optican LM, Cook JD, Robinson DA (1976) Slow saccades in spinocerebellar degeneration. Arch Neurol 33:243-251.

Zipser D, Andersen RA (1988) A back-propagation programmed network that simulates response properties of a subset of posterior parietal neurons. Nature 331:679-684. 